\title{
Effects of geometric shape on the hydrodynamics of a self-propelled flapping foil
}

\author{
Xing Zhang, Saizhen Ni, Shizhao Wang, and Guowei He ${ }^{a)}$ \\ LNM, Institute of Mechanics, Chinese Academy of Sciences, Beijing 100190, China
}

(Received 17 March 2009; accepted 21 September 2009; published online 22 October 2009)

\begin{abstract}
The hydrodynamics of a free flapping foil is studied numerically. The foil undergoes a forced vertical oscillation and is free to move horizontally. The effect of chord-thickness ratio is investigated by varying this parameter while fixing other ones such as the Reynolds number, the density ratio, and the flapping amplitude. Three different flow regimes have been identified when we increase the chord-thickness ratio, i.e., left-right symmetry, back-and-forth chaotic motion, and unidirectional motion with staggered vortex street. It is observed that the chord-thickness ratio can affect the symmetry-breaking bifurcation, the arrangement of vortices in the wake, and the terminal velocity of the foil. The similarity in the symmetry-breaking bifurcation of the present problem to that of a flapping body under constraint is discussed. A comparison between the dynamic behaviors of an elliptic foil and a rectangular foil at various chord-thickness ratios is also presented. (C) 2009 American Institute of Physics. [doi:10.1063/1.3251045]
\end{abstract}

\section{INTRODUCTION}

Flapping wings or fins are commonly used by birds, insects, fishes, and some microair vehicles (MAVs) to generate propulsive forces. To understand the mechanism of thrust generation, many experimental and numerical studies on flapping foils have been conducted, such as in Refs. 1-6. It is found that a reversed Karman vortex street in the wake of the flapping body is the fundamental feature associated with thrust generation. In most of the studies mentioned above, the foil is placed in a steady stream and its movement in the streamwise direction is prohibited. The flapping motion of the foil is usually prescribed, either heaving or pitching or the combination of these two. However, the conditions in these studies are completely different from those in selfpropelled biolocomotions. In other words, the body velocity in real biolocomotion is allowed to change in response to the variation of the forward force. To our knowledge, only a few papers can be found in the study of fundamental hydrodynamics of a self-propelled flapping foil. In the experiment by Vandenberghe et al., ${ }^{7,8}$ it is shown that an axle-mounted blade can spontaneously rotate when flapped vertically in a fluid. In the numerical investigations, Blackburn et al..$^{9}$ simulated a bluff-body propulsion produced by a combined rotary and translational oscillation; Alben and Shelley ${ }^{10}$ studied the locomotion generation by a free flapping body through symmetry breaking and vortex interactions; Alben ${ }^{11}$ proposed an efficient numerical method to compute the coupled flow-body dynamics. Recently, $\mathrm{Lu}$ and $\mathrm{Liao}^{12}$ numerically predicted the critical Reynolds number (which is based on the flapping frequency and amplitude) of the problem proposed in Ref. 10. Furthermore, there are some studies on the self-propelled fish swimming where the

\footnotetext{
a) Author to whom correspondence should be addressed. Telephone: +86-10-82543969. Fax: +86-10-82543977. Electronic mail: hgw@1nm.imech.ac.cn.
}

prescribed time-dependent motion forms a backwardtraveling wave. ${ }^{13-15}$

In this paper, we consider the effect of geometric shape on the hydrodynamics of a self-propelled foil. This study is motivated by the quest to understand the relation between the morphology of wings (fins) and their hydrodynamic performance. A better understanding of this subject will be helpful to the design of MAVs or autonomous underwater vehicles. In this study, the foil is forced to flap up and down in a sinusoidal fashion and free to move horizontally. The moving unstructured Chimera mesh technique developed in Refs. 16 and 17 is an efficient approach for the present problem because the meshes need not to be regenerated when the foil moves around freely. Thus, this numerical method is adopted in this study. Numerical simulations are performed on the elliptic and rectangular foils with the chord-thickness ratios (CRs) ranging from 1:1 to 100:1, while other parameters such as the Re number, the density ratio, and the flapping amplitude are fixed.

The organization of the paper is as follows. The governing equations are given in Sec. II. The computational method is then described in Sec. III. The numerical observations are presented in Sec. IV. Finally, Sec. V concludes the article with a summary and discussion.

\section{A MODEL PROBLEM}

The fluid motion is governed by the two-dimensional incompressible Navier-Stokes equations,

$$
\begin{aligned}
& \frac{\partial \mathbf{u}}{\partial t}+\mathbf{u} \cdot \nabla \mathbf{u}=-\nabla p+\frac{1}{\operatorname{Re}} \nabla^{2} \mathbf{u}, \\
& \nabla \cdot \mathbf{u}=0,
\end{aligned}
$$

where the Reynolds number Re is defined as 


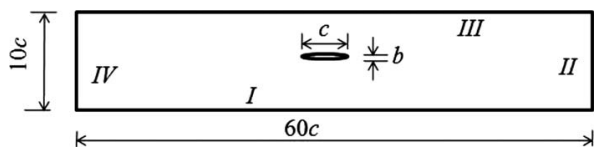

FIG. 1. A schematic representation of the computational domain. $c$ is the chord length of the foil and $b$ is the thickness.

$$
\operatorname{Re}=A \omega c / \nu .
$$

Here $c$ is the chord length of the foil and $\nu$ is the dynamic viscosity of the fluid. $A$ and $\omega$ are the amplitude and the angular frequency respectively of the sinusoidal oscillation. The characteristic length, velocity, and time used to nondimensionalize Eqs. (1) and (2) are $c, A \omega$, and $c /(A \omega)$, respectively.

The motion of the foil in the horizontal direction is governed by Newton's law,

$$
M \frac{d \bar{u}_{b}}{d t}=F_{x}
$$

Here $\bar{u}_{b}$ is the dimensionless horizontal velocity of the foil. $F_{x}$ is the dimensionless horizontal force evaluated from the solution of Eqs. (1) and (2) by integrating on the surface of the foil. $M$ is the dimensionless mass of the foil defined as

$$
M=\frac{\rho_{b} S}{\rho_{f} c^{2}},
$$

where $\rho_{b}$ and $\rho_{f}$ are the density of the foil and the fluid, respectively, and $S$ is the area of the foil.

The vertical motion is prescribed as

$$
y_{b}=A \sin (\omega t) \text {. }
$$

Using the aforementioned characteristic length and time scales, this formula can be converted to a dimensionless form as

$$
\bar{y}_{b}=\bar{A} \sin (\bar{t} / \bar{A}),
$$

where the bar symbol denotes dimensionless variables, i.e.,

$$
\begin{gathered}
\bar{y}_{b}=y_{b} / c, \\
\bar{A}=A / c, \\
\bar{t}=A \omega t / c .
\end{gathered}
$$

\section{COMPUTATIONAL METHOD}

This study employs an unstructured Chimera grid method to solve the Navier-Stokes equations. This approach is very flexible in the sense that it allows the use of multiple unstructured meshes that overlap each other to simulate the objects of arbitrary shapes and arbitrary relative motions. For the technical details of this numerical methodology and some benchmark validations, please refer to Refs. 16 and 17.

A schematic representation of the computational domain in the present problem is given in Fig. 1. On the four boundaries (I-IV) of the domain and the surface of the foil, nonslip

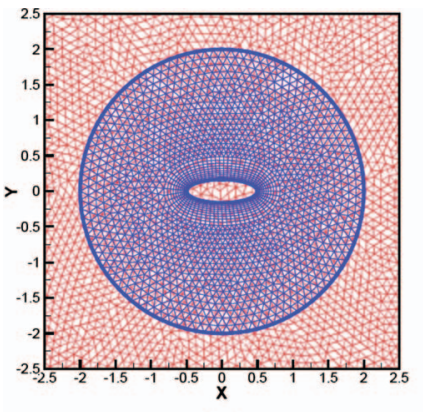

(a)

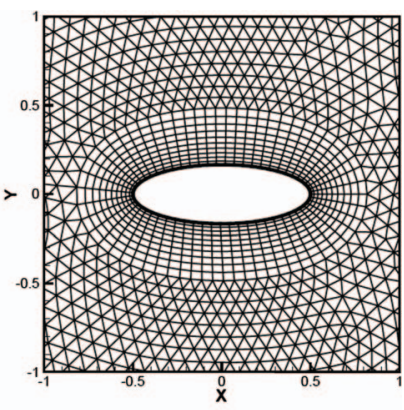

(b)
FIG. 2. (Color) Meshes for the case of $C R=3$. (a) Meshes near the overlapping region (red line: background mesh; blue line: moving mesh; thick blue line: boundary of the moving domain); (b) local mesh around the foil surface where rectangular cells are used to capture the flow phenomenon in the boundary layer.

boundary conditions are applied. On the internal boundaries between the overlapping grids, interpolation of velocity and pressure are used to couple the solutions.

The present domain size of $60 c \times 10 c$ is chosen as a compromise to save computational time and meanwhile avoid too much blocking effect from the boundaries. The local meshes for an elliptic foil at the CR equals to 3 are shown in Fig. 2. The number of mesh points that are deployed on the surface of the foil is 60 . For the cases of larger CRs (slender foils), this number is increased accordingly. These extra grid points are clustered near the leading and trailing edges where the large gradients of flow quantities exist. The distance from the first off-wall point to the surface is 0.02 . The number of mesh points in each case is determined so that the boundary layer from the body is well resolved. The time step used in the simulation is 0.005 and the maximum Courant-Friedrichs-Lewy (CFL) number is around 0.2. This maximum CFL number is defined as

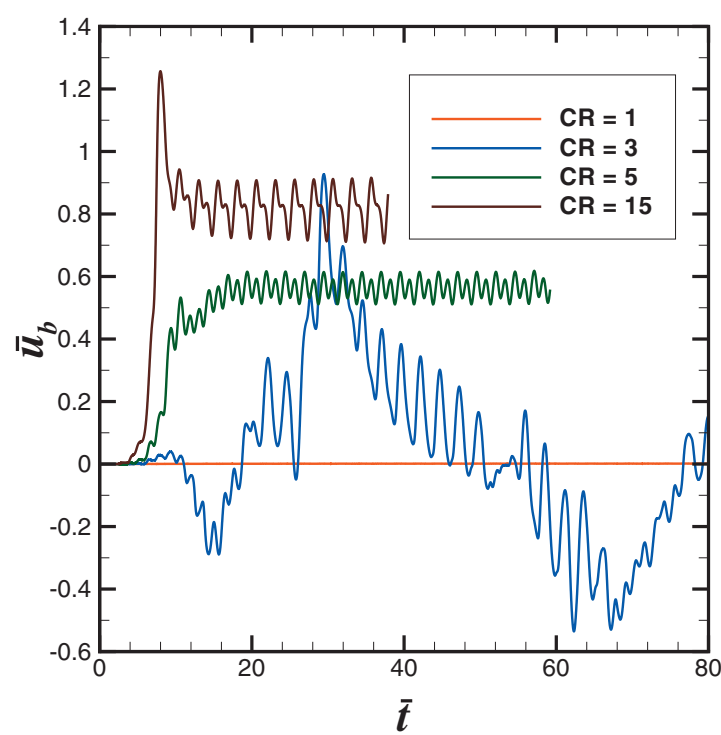

FIG. 3. (Color) The time history of the horizontal velocities for elliptic foils at various CRs. Red: $\mathrm{CR}=1$ (no symmetry breaking); blue: $\mathrm{CR}=3$ (chaotic motion); green: $\mathrm{CR}=5$ (unidirectional motion); brown: $\mathrm{CR}=15$ (unidirectional motion). 


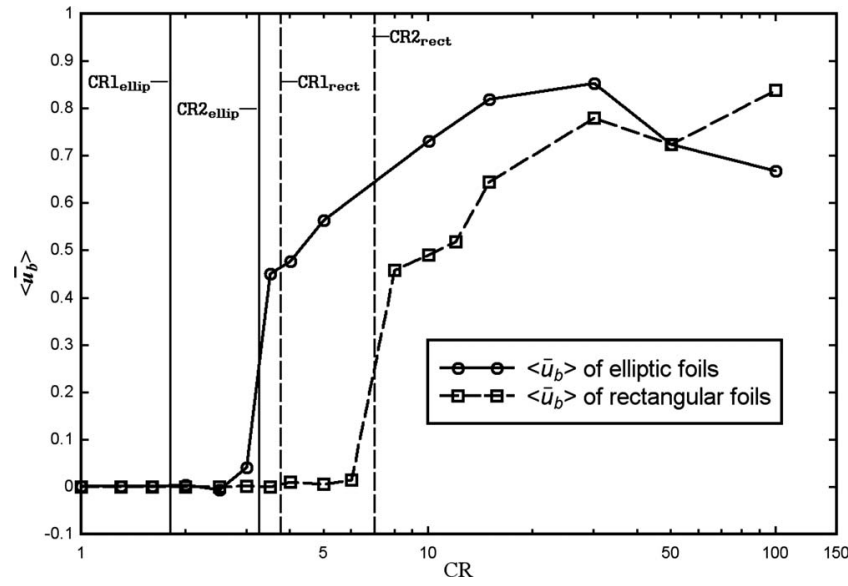

FIG. 4. Time averaged horizontal velocity of the foils vs CR. The two boundaries between the three different scenarios are labeled. The velocities are averaged after a periodic steady state is reached for the unidirectional motions; while for other scenarios, they are averaged after ten periods of foil oscillations to minimize the influence of initial condition.

$\mathrm{CFL}_{\max }=\Delta t(|u| / \Delta h)_{\max }$, where $|u|$ is the velocity magnitude and $\Delta h$ is the mesh size in each cell. After some gird size and time step independent tests, it is confirmed that the mesh resolution used in this study is fine enough and the time step is sufficiently small to obtain an accurate solution.

\section{NUMERICAL OBSERVATIONS}

There are four important dimensionless parameters in our problem: the Reynolds number Re, the density ratio $\rho_{b} / \rho_{f}$, the oscillation amplitude $A / c$, and the CR $c / b$ (with $b$ being the thickness of the foil). In this work, the following parameters are used: $\operatorname{Re}=200, \rho_{b} / \rho_{f}=4, A / c=0.4$, and $c / b=1-100$. The first three parameters are fixed (except where otherwise stated) while the CR varies in the range of 1-100. Simulations are performed for both the elliptic and rectangular foils. The observations from these simulations are summarized as follows.

\section{A. Elliptic foil}

The time history of the horizontal velocities for $\mathrm{CR}=1$, 3, 5, and 15 are plotted in Fig. 3. From this figure, it is seen that three different scenarios can be identified with the variation of $\mathrm{CR}$, namely, no symmetry breaking $(\mathrm{CR}=1)$, chaotic motion $(\mathrm{CR}=3)$, and unidirectional locomotion $(\mathrm{CR}=5$ and 15$)$.

In Fig. 4, the time averaged velocity is plotted as a function of CR. The two boundaries between the three scenarios aforementioned are labeled in this figure as CR1 and CR2, respectively. It is found through numerical experiments that for an elliptic foil, CR1 and CR2 are located in the narrow band of $1.6-2.0$ and 3.0-3.5, respectively. It is also noted that if we increase the CR within the region of CR $>$ CR2, the vortex structure in the wake does not change qualitatively although the averaged terminal velocity varies (this will be shown latter).

To explore the cause of the different dynamics behaviors in the three scenarios above, the flow fields of some typical

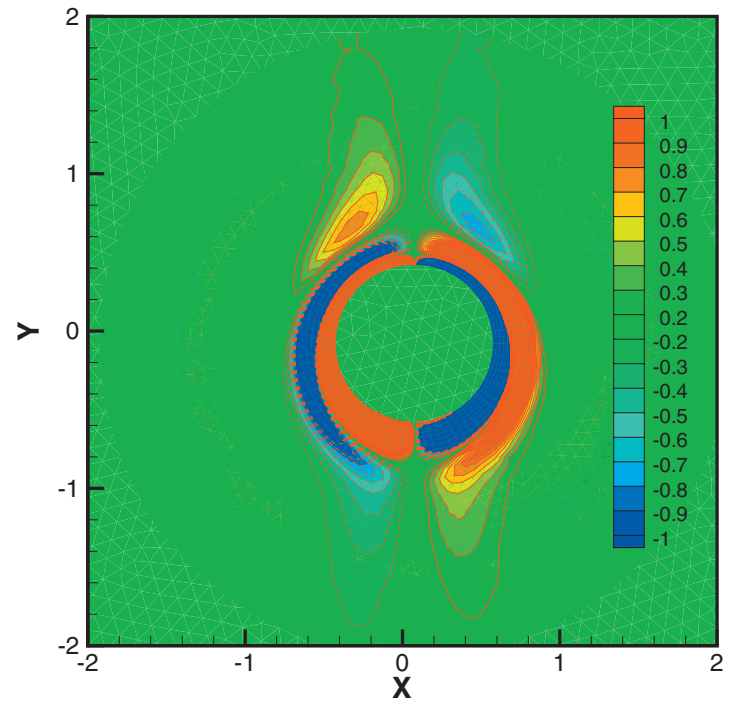

FIG. 5. (Color) Vorticity contours of an elliptic foil $(\mathrm{CR}=1)$ at $\bar{t}=200$. Despite a minor deviation of the symmetric axle from its initial position at $\mathrm{x}=0$, the left-right symmetric pattern of the vorticity contours is kept [URL: http://dx.doi.org/10.1063/1.3251045.1] (Enhanced online).

cases are illustrated here. The first case corresponds to the symmetric and stable state (scenario I), the second one corresponds to the chaotic state (scenario II), while the third and the fourth ones correspond to the unidirectional locomotion (scenario III).

The first case is $C R=1$, where the foil is actually a circular cylinder. It is found that no effective locomotion is triggered by numerical perturbations (e.g., machine error and mesh asymmetry). It is seen that a left-right symmetric pattern persists at $\bar{t}=200$ (see the vorticity contours in Fig. 5). It is also found that the horizontal velocity does not decay to machine zero after a long time of integration but its magnitude becomes very small (less than $10^{-3}$ ). Further investigation confirms that this small velocity is caused by the left-right mesh asymmetry and it can be further reduced to $O\left(10^{-4}\right)$ by using a mesh of better symmetric property. To test the stability of this left-right symmetric state, we also impose a large initial perturbation of 0.01 on the
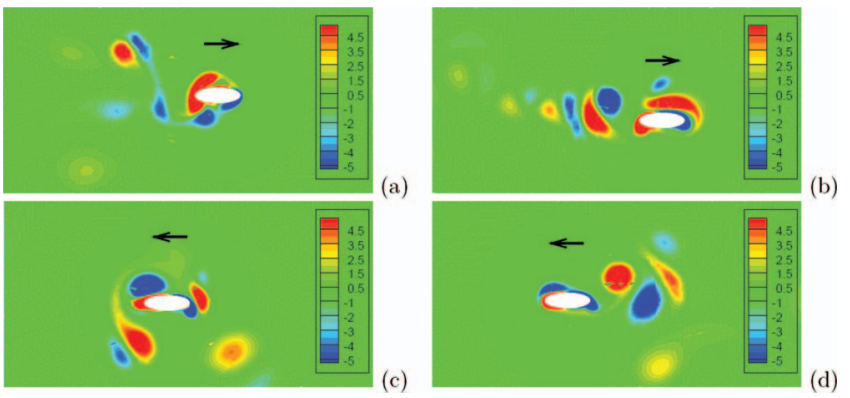

FIG. 6. (Color) Instantaneous vorticity contours of an elliptic foil $(\mathrm{CR}=3)$ : (a) $\bar{t}=30$, (b) $\bar{t}=50$, (c) $\bar{t}=60$, and (d) $\bar{t}=70$. The black arrows indicate the direction of the velocity of the foil. The formation of vortex pairs can be seen in (a), (c), and (d) [URL: http://dx.doi.org/10.1063/1.3251045.2] (Enhanced online). 


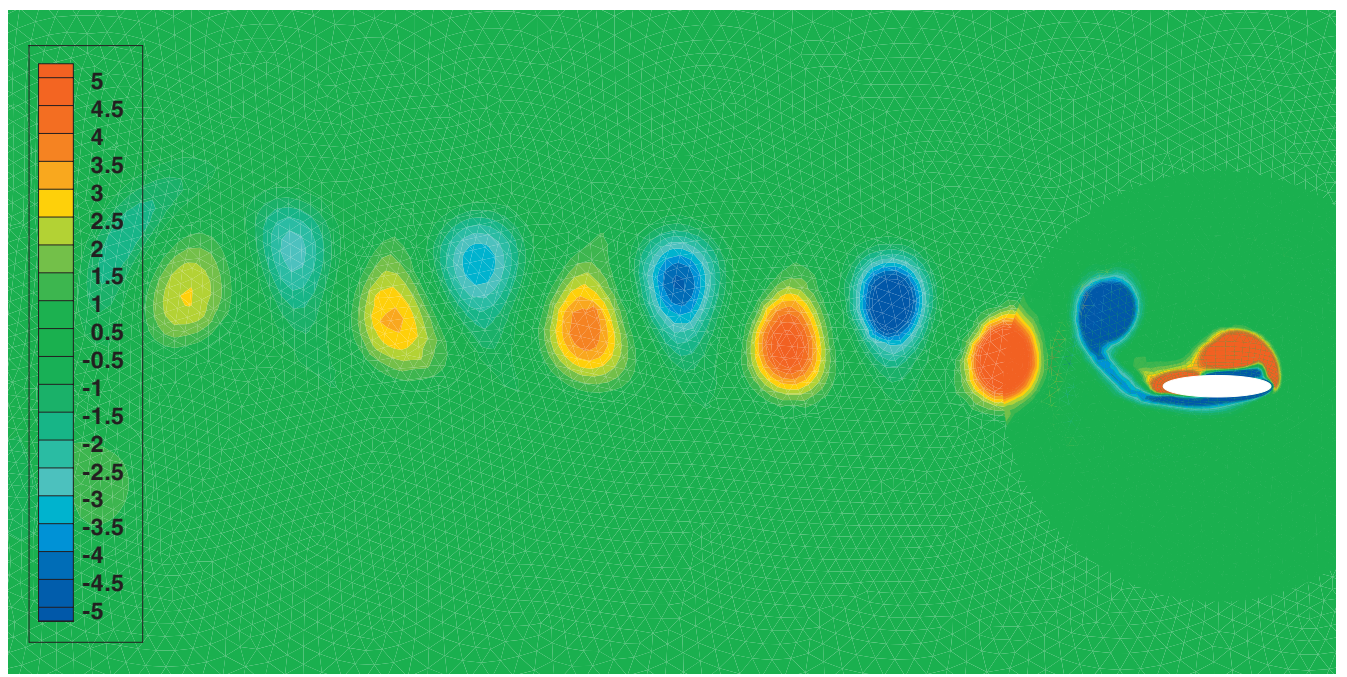

FIG. 7. (Color) The reversed Von Karman vortex street behind an elliptic foil of $\mathrm{CR}=5$. The large vortical structures propagate almost horizontally to the left as the foil moves toward the right [URL: http://dx.doi.org/10.1063/1.3251045.3] (Enhanced online).

horizontal velocity of the foil. It is found that terminal velocity is almost the same as the one that is obtained without the perturbation.

The second case is $\mathrm{CR}=3$. In this case, the symmetric pattern in the flow field is lost at $\bar{t}=4$. Soon after symmetry breaking, a vortex dipole (pairs) begins to emerge at one edge of the foil. This dipole provides the propulsive force to accelerate the foil toward one direction (this direction depends on the initial conditions and is found to be the left in this case). From the time history of the horizontal velocity that is shown in Fig. 3, it is seen that the foil moves back and forth in a chaotic manner and no unidirectional locomotion is achieved after a long time of simulation (the averaged velocity is very close to zero). The chaotic dynamic behavior of the foil is associated with the arrangement of the vortices and the complex interactions among them (see Fig. 6).

The third case is $\mathrm{CR}=5$. The starting procedure (including the symmetry breaking and the acceleration by vortex dipoles) in this case is similar to that in the case of $\mathrm{CR}=3$. However, soon after the starting, a large vortical structure is found to propagate almost horizontally to the left (the choice of direction depends on the initial condition). This vortical structure possesses an alternating asymmetry about the center line (see Fig. 7).

This pattern is also termed as the reversed Von Karman vortex street in some textbooks. Clearly, a unidirectional locomotion is achieved in this case. The averaged terminal velocity is found to be 0.56 from the time history plot of the horizontal velocity (see Fig. 3).

The fourth case is $C R=15$. The qualitative nature of the vortex structure in this case is the same as that of $\mathrm{CR}=5$. The only difference lies in the angle of propagation of the vortex structure in the wake (see Fig. 8). In this case, the "dipoles" are arranged along an oblique axis which is below the center line ("below" or "above" depends on the initial per-

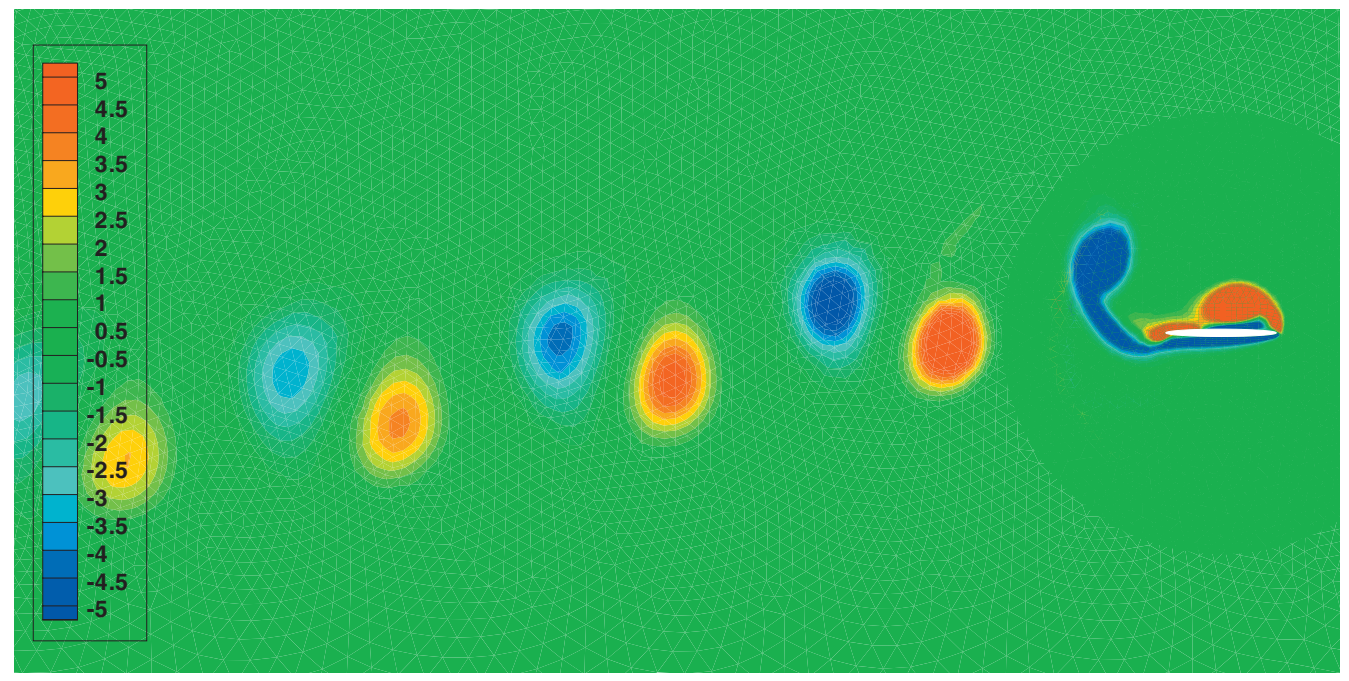

FIG. 8. (Color) The reversed Von Karman vortex street behind an elliptic foil of $\mathrm{CR}=15$. The large vortical structures propagate to the left obliquely (deflected downwards) as the foil moves toward the right [URL: http://dx.doi.org/10.1063/1.3251045.4] (Enhanced online). 


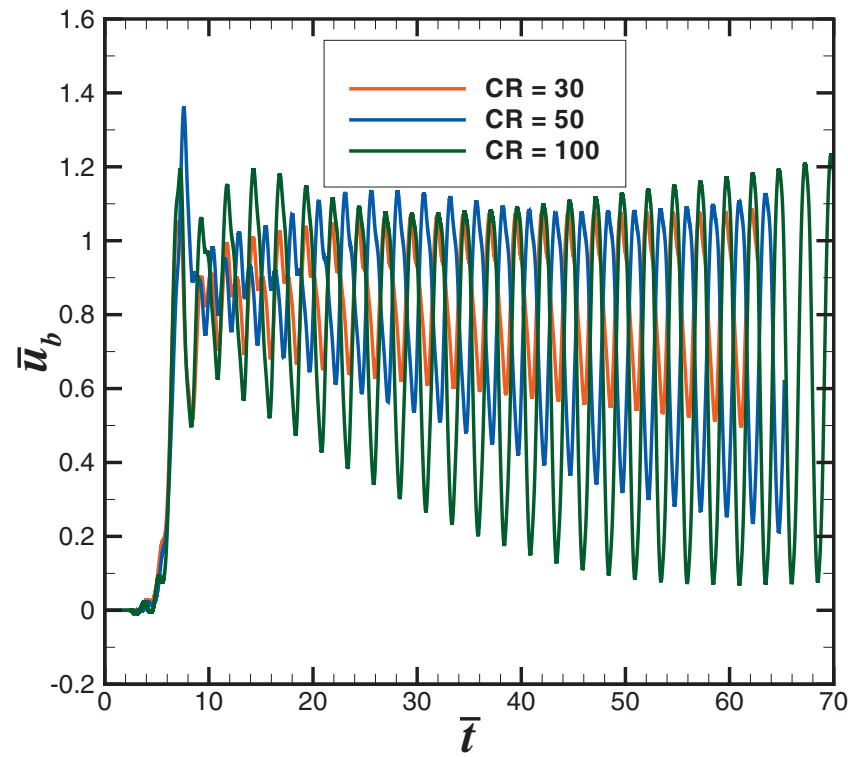

FIG. 9. (Color) A comparison of the time histories of the horizontal velocity of elliptic foils at three different CRs. Red color denotes the case of $\mathrm{CR}=30$, blue color denotes the case of $\mathrm{CR}=50$, and green color denotes the case of $\mathrm{CR}=100$.

turbation in the simulation). The terminal averaged velocity is found to be 0.82 , which is much larger than that in the case of $\mathrm{CR}=5$.

With the further increase in $\mathrm{CR}$ (e.g., $\mathrm{CR}=30,50$, or $100)$, the vortex structure remains to be very similar to that of $\mathrm{CR}=15$. The terminal averaged velocity is found to first increase and then decrease with the increase in CR (see Fig. 4). The maximal terminal velocity is 0.85 at $C R=30$ among the cases we studied. It is also observed that when the CR becomes very large (e.g., $\mathrm{CR}=50$ or 100 ), large velocity fluctuation is produced (see Fig. 9). This large fluctuation in the horizontal velocity is probably due to rather small mass in such thin foils.

Recalling the previous discussions on the cases of lower $\mathrm{CRs}$, we can conclude that the $\mathrm{CR}$ has two contradicting effects on the system. Foils with smaller CR (thicker foils) have a larger added mass, i.e., more fluid needs to be pushed out of the way for it to move forward. Thus the foils with larger CR (thinner foils) move more easily than the thicker ones. On the other hand, if the foil is too thin then its mass becomes too small. Large velocity oscillations can be produced and the terminal velocity is even reduced.

From the numerical results above, it is seen that some important features in the hydrodynamics can be influenced by the CR. First of all, CR affects the stability and the symmetry-breaking bifurcation. Below the critical ratio CR1, the left-right symmetric state is stable and symmetry breaking does not occur. However, the value of CR1 is not a constant and may depend on other parameters such as the Reynolds number, the density ratio, and the oscillating amplitude. For example, the circular cylinder $(\mathrm{CR}=1)$ produces a stable and symmetric pattern at $A / c=0.4$, but as the oscillation amplitude increases, other flow pattern emerges. It is shown in Figs. 10(a) and 10(b) that back-and-forth and unidirectional motions can be achieved at $A / c=0.8$ and $A / c=1.0$, respectively.

The CR can also affect the critical Reynolds number of the symmetry breaking. It is found by some researchers that the critical Reynolds number decreases as the CR increases. ${ }^{10}$

The stability and the symmetry-breaking bifurcation in this problem are still not very well understood. What is observed here seems to share some similarity with the symmetry-breaking bifurcation of an oscillating body that is described in Ref. 18. In that study, a circular cylinder $(\mathrm{CR}=1)$ is forced to oscillate in one direction and is constrained in the perpendicular one. The results in Ref. 18 are summarized in a diagram (Fig. 4 of Ref. 18) in which the parametric space $(\mathrm{Re}, A / c)$ is categorized into different flow regimes [in Ref. 18 a Keulegan-Carpenter (KC) number which is defined as $2 \pi A / c$ is used in the diagram]. It is found that for the case of $C R=1$, the point $(200,0.4)$ is located in the regime of "stable and symmetric pattern" in the diagram while the points $(200,0.8)$ and $(200,1.0)$ are not. This evidence suggests that if the "left-right symmetry"

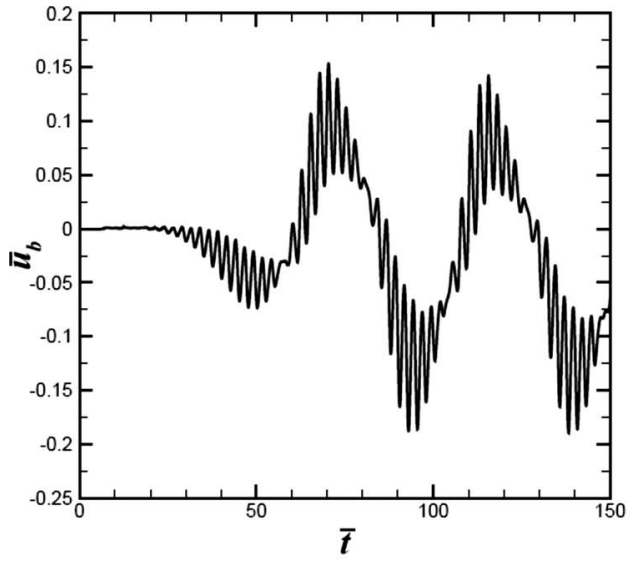

(a)

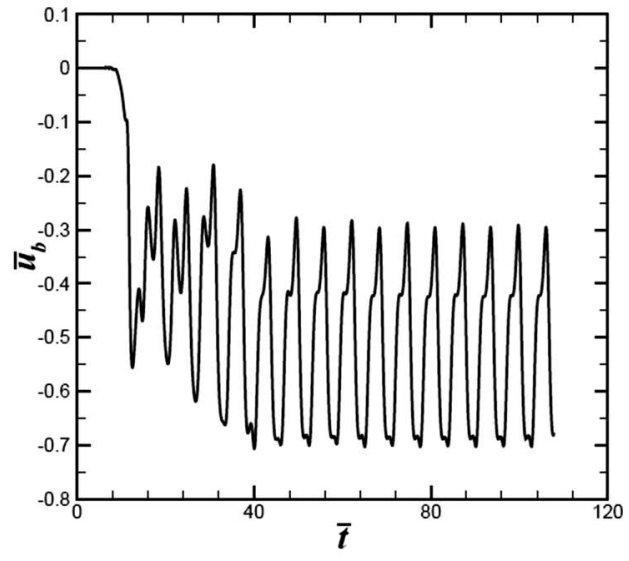

(b)

FIG. 10. Time histories of the horizontal velocity of an elliptic foil at $\mathrm{CR}=1$ (circular cylinder) but with lager oscillating amplitudes. (a) is for $A / c=0.8$ (chaotic motion) and (b) is for $A / c=1.0$ (unidirectional locomotion). Symmetry breaking does occur in both cases. 
is a stable state for a horizontally constrained it is also a stable state for a freely moving one (at least when small perturbations are considered). Conversely, if the left-right symmetry is unstable when the foil is horizontally constrained, then it is also unstable when the foil is free to move. To check the validity of this conjecture, we also conduct some additional tests on the cases of $\mathrm{CR}=3$ and $\mathrm{CR}=5$ by constraining the foil in the horizontal direction. It is found that an asymmetric vortical pattern develops naturally after some periods of oscillation. The asymmetry in the horizontally constrained state was also studied in Ref. 11. It was also found there that the flow asymmetry happened at nearly the same parameter value in both the constrained and freely moving cases.

The arrangement of the vortices is another important feature of the flow that is closely related to the CR. It is found that a thick body (e.g., $C R=3$ ) cannot easily shake off the vortices that are shed during the previous strokes. These vortices tend to circulate around the body and give rise to complicated interactions among them. A slender body $(\mathrm{CR}>\mathrm{CR} 2)$ can easily organize the vortices into large structures (oblique dipoles or staggered vortex street). These vortical structures generate large propulsive force on the body and the body is then accelerated quickly to gain the forward velocity. A large forward velocity in turn makes it easier to shake off the vortices from the previous strokes. The vortex shedding at an opportune moment, the formation of large structures, and the coupling between the hydrodynamics and the body dynamics are the three dominant factors in a smooth transition to unidirectional locomotion.

Finally, the CR also affects the terminal velocity (if a unidirectional locomotion can be generated). The trend that the terminal velocity first increases then decreases with the increase in CR can be clearly seen in Fig. 4. It is found that a maximal velocity of 0.85 is achieved near the CR of 30 .

The different motions observed with the variation of CR are mainly attributed to the following two effects: (1) the increased strength of vorticity shed from a sharper edge (higher CR) and (2) the increased form drag on a thicker body (lower CR). Furthermore, since the self-propelled system enables the coupling between the flow and the body dynamics, the change in mass of the foil with the variation of CR should also be taken into consideration.

\section{B. Rectangular foil}

Rectangular shape is of interest since it is the real geometry that is adopted in some experiments, such as in Refs. 7 and 8 . Furthermore, the presence of sharp corners in the rectangular body may induce more intensive vorticity and this is very different from the elliptic one. In this study, the thickness-chord ratios previously assigned to the elliptic foil are chosen and the simulations are also performed on the rectangular foils. From the results, it is found that as we increase the $\mathrm{CR}$, the trend in the flow pattern, and body dynamics is quite similar for both shapes. Most of the qualitative descriptions in Sec. IV A (such as the symmetric breaking bifurcation, the three distinct scenarios and the vortical patterns in the wake) are also valid in case of the rect- angular foils. However, differences can still be found between them in the curves of averaged velocity versus CR (see Fig. 4). Some differences are highlighted here.

Results on the critical CRs CR1 and CR2 show that the rectangular foils tend to transit at larger critical CR than the elliptic foils do (both from the symmetric stable state to the chaotic motion and from the chaotic motion to the unidirectional locomotion). It is found that elliptical foils have a narrower "chaotic" locomotion region than the rectangular foils.

From Fig. 4, it is also seen that for most CRs that are studied in this paper $(\mathrm{CR}<50)$, an elliptic foil moves faster than a rectangular foil. This trend is not seen for very slender foils, e.g., at $\mathrm{CR}=50$ the velocities are almost the same while at $\mathrm{CR}=100$ a rectangular foil even moves faster than an elliptic one. For the elliptic foils, the terminal velocity first increases and then decreases with the increase in CR and a maximum terminal velocity is achieved at $\mathrm{CR}=30$. However, for the rectangular foils, such simple trend in terminal velocity is not found. Actually, on the velocity-CR curve of the rectangular foil, a local minimum value is found at $\mathrm{CR}=50$ where the two velocity curves intersect (i.e., the foils of the two shapes have the same velocity). In the range of $\mathrm{CR}$ that is studied in this paper $(1<\mathrm{CR}<100)$, the largest terminal velocity for a rectangular foil is reached at $\mathrm{CR}=100$. This velocity is very close to the maximal velocity of an elliptic foil at $\mathrm{CR}=30$. Due to the concern of numerical inaccuracy, we do not perform any simulations on foils with CR larger than 100 .

To further analyze the reason behind the difference in terminal velocity from a rectangular foil and an elliptic foil, we need to consider two factors that affect the generation of thrust and form drag. First, a rectangular foil induces relatively higher vorticity concentration near the body (thus larger pressure difference) due to the presence of sharp corners. Second, a rectangular foil only utilizes pressure differences on the two sides (left-facing and right-facing) while an elliptic foil utilizes pressure differences in the whole surface. The competition of these two effects eventually determines the difference in terminal velocity.

\section{SUMMARY AND DISCUSSION}

We study the effects of geometric shape on the hydrodynamical characteristics of a foil that is forced to oscillate vertically and free to move horizontally. It is found that the CR can affect the stability and the symmetry breaking of the system. A threshold of the CR (below which the bifurcation does not occur) is found to be somewhere between 1.6 and 2.0 for an elliptic foil and between 3.5 and 4.0 for a rectangular foil. This value is based on the simulations when other parameters are fixed (i.e., $\operatorname{Re}=200, \rho_{b} / \rho_{f}=4$, and $A / c=0.4$ ). The CR also affects the final state of motion if the symmetry breaking occurs. Thicker foils give rise to a back-and-forth (chaotic) motion; slender foils produce the structure of stagger vortex street or oblique dipoles in the wake and generate a unidirectional locomotion. We also found that the symmetry-breaking bifurcation observed in this study shares 
some similarity with that of a constrained oscillating body which is studied in Ref. 18 although no mathematical explanation is currently available.

Some implications of this study on biolocomotion are summarized below. First, it is easier and faster for a slender foil to break the symmetry and transit to a unidirectional motion than for a thick foil. Second, an elliptic foil with a CR between 15 and 30 tends to operate in the optimized Strouhal number. From Ref. 3 it is known that the Strouhal number [which is defined as $(A \omega) /(\pi U)]$ in animal cruising is tuned to be within a narrow band between 0.2 and 0.4 for the reason of energy efficiency. In our study, it is found that for an elliptic foil of $\mathrm{CR}=5$, the Strouhal number is 0.57 , while for $\mathrm{CR}=15$, the Strouhal number becomes 0.39 . This number can be brought down to 0.37 if the CR reaches 30 . While for a rectangular foil, it is found that such an optimized Strouhal number (approximately 0.38) is achieved at $\mathrm{CR}=100$. An interesting finding is that the optimized Strouhal number is achieved at a much larger CR on the rectangular foils than that on the elliptic foils.

To explore the whole four-dimensional space ( $\mathrm{Re}, A / c$, $\rho_{b} / \rho_{c}$, and $\left.c / b\right)$ will be very helpful to the understanding of the physics in this problem. However, an exhaustive parametric study is still very time consuming based on our current computing capability. It would be a good direction in future endeavors. Another avenue for further research is the role of asymmetry in the self-propelled flapping system. In the present work, the Strouhal numbers are only compared among the foils of left-right symmetric shapes. It is well known that the asymmetry in the foil shape (e.g., sharp trailing edge) can enhance the thrust generation significantly. ${ }^{19}$ Besides that the asymmetry in the flapping motion (e.g., pitching) is also very important in some biolocomotion problems, such as motions starting from rest.

\section{ACKNOWLEDGMENTS}

This work was supported by the Chinese Academy of Sciences under the Innovative Projects "Multi-scale modeling and simulation in complex systems" (Grant No. KJCX-SW-L08) and "Mathematical modeling of complex system" (Grant No. KJCX3-SYW-S01), the
National Basic Research Program of China (973 Program) under Project No. 2007CB814800, and the National Natural Science Foundation of China under Project Nos. 10325211, 10628206, 10732090, and 10872201.

${ }^{1}$ M. M. Koochesfahani, "Vortical patterns in the wake of an oscillating airfoil," AIAA J. 27, 1200 (1989).

${ }^{2}$ P. Freymuth, "Thrust generation by an airfoil in hover modes," Exp. Fluids 9, 17 (1990).

${ }^{3}$ M. S. Triantafyllou, G. S. Triantafyllou, and R. Gopalkrishnan, "Wake mechanics for thrust generation in oscillating foils," Phys. Fluids A 3, 2835 (1991)

${ }^{4}$ K. D. Jones, C. M. Dorhring, and F. M. Platzer, "Experimental and computational investigation of the Knoller-Betz effect," AIAA J. 36, 1240 (1998).

${ }^{5}$ K. Streitlien and G. S. Triantafyllou, "On thrust estimates for flapping foils," J. Fluids Struct. 12, 47 (1998).

${ }^{6}$ C. S. J. Lai and F. M. Platzer, "Jet characteristics of a plunging airfoil," AIAA J. 37, 1529 (1999).

${ }^{7}$ N. Vandenberghe, J. Zhang, and S. Childress, "Symmetry breaking leads to forward flapping flight," J. Fluid Mech. 506, 147 (2004).

${ }^{8} \mathrm{~N}$. Vandenberghe, S. Childress, and J. Zhang, "On unidirectional fight of a free flapping wing," Phys. Fluids 18, 014102 (2006).

${ }^{9}$ H. M. Blackburn, "Bluff-body propulsion produced by combined rotary and translational oscillation," Phys. Fluids 11, 4 (1999).

${ }^{10} \mathrm{~S}$. Alben and M. Shelley, "Coherent locomotion as an attracting state for a free flapping body," Proc. Natl. Acad. Sci. U.S.A. 102, 11163 (2005).

${ }^{11}$ S. Alben, "An implicit method for coupled flow-body dynamics," J. Comput. Phys. 227, 4912 (2008).

${ }^{12} \mathrm{X}$. Y. Lu and Q. Liao, "Dynamic responses of a two-dimensional flapping foil motion," Phys. Fluids 18, 098104 (2006).

${ }^{13}$ J. Carling, T. L. Williams, and G. Bowtell, "Self-propelled anguilliform swimming: simultaneous solution of the two-dimensional Navier-Stokes and Newton's laws of motion," J. Exp. Biol. 201, 3143 (1998).

${ }^{14}$ A. Leroyer and M. Visoneau, "Numerical methods for RANSE simulations of a self-propelled fish-like body," J. Fluids Struct. 20, 975 (2005).

${ }^{15}$ S. Kern and P. Koumoutsakos, "Simulations of optimized anguilliform swimming," J. Exp. Biol. 209, 4841 (2006).

${ }^{16} \mathrm{X}$. Zhang, "Computation of viscous incompressible flow using pressure correction method on unstructured Chimera grid," Int. J. Comput. Fluid Dyn. 20, 637 (2006).

${ }^{17}$ X. Zhang, S. Z. Ni, and G. W. He, "A pressure-correction method and its applications on an unstructured Chimera grid," Comput. Fluids 37, 993 (2008).

${ }^{18}$ H. Dutsch, F. Durst, S. Becker, and H. Lienhart, "Low-Reynolds-number flow around an oscillating circular cylinder at low Keulegan-Carpenter numbers," J. Fluid Mech. 360, 249 (1998).

${ }^{19}$ D. D. J. Chandar and M. Damodaran, "Computational study of unsteady low-Reynolds-number airfoil aerodynamics using moving overlapping meshes," AIAA J. 46, 429 (2008). 